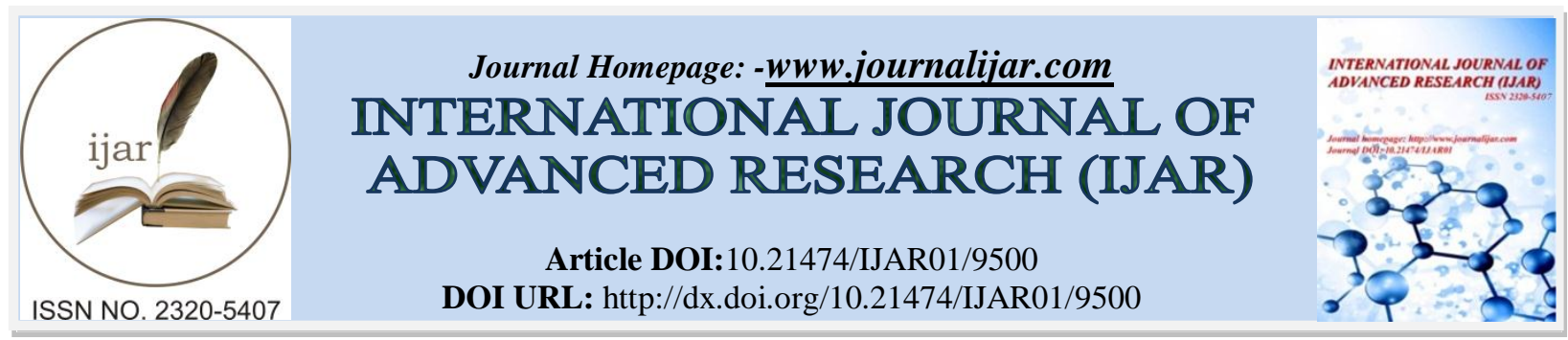

RESEARCH ARTICLE

\title{
EVALUATION OF AWARENESS AND ATTITUDE OF RADIOLOGICAL TECHNOLOGIST TOWARDS RADIATION SAFETY IN DHAKA CITY OF BANGLADESH.
}

Helal Uddin ${ }^{1}$, Tanzila Parvin ${ }^{2}$, SM Muraduzzaman² And Faridul Alam'.

1. Department of Radiology \& Imaging Technology, Bangladesh University of Health Sciences, Dhaka, Bangladesh.

2. Department of Biomedical Engineering \& Medical Physics, Bangladesh University of Health Sciences, Dhaka, Bangladesh.

\section{Manuscript Info}

n......................

Manuscript History

Received: 06 June 2019

Final Accepted: 08 July 2019

Published: August 2019

Key words:-

Radiation Safety, ALARA, Awareness, Knowledge , Performance , Practice , Ionizing Radiation , Radiation protection.

\section{Abstract}

Radiation safety culture in health care considers radiation protection of patients, health workers and the general public. Radiation protection is the science and art of protecting people and the environment from the harmful effects of ionizing radiation. Ionizing radiation in medical imaging is one of the powerful diagnostic tools, and accurate knowledge of radiation protection will affect the radiological technologists' safety behaviors during practice. This is a preliminary descriptive study aimed to evaluate radiological technologist knowledge and performance towards radiation protection during their working time. Total of 75 radiology technologists who work in various hospitals were participated in this study and data was collected through well-structured pretested self-administered questionnaire. Among 75 radiology technologist responded to this study, there were $75 \%$ male and $25 \%$ female and education qualification for most of them were Diploma $(80.7 \%)$ level. Moreover the study did not find any relation between level of education of participants and work expertise with their knowledge around necessity performance of periodical examination. According to data analysis, there was no significant relation between awareness of radiation safety, performance and work experience. In this study there were $(75 \%)$ male and $(25 \%)$ female and most of them had no bachelor degree (about $80 \%$ ). Regarding radiation protection and awareness, majority of the participants (98.7\%) only know that doors and walls should be well shielded, they should wear personal monitoring devices during work time to monitor the individual dose limit and their devices used to check periodically providing assurance that dose limits are not exceeded. But most of radiology technologists are very unconscious about patient safety. Only $10 \%$ of respondents have some knowledge on ALARA Principle and ionizing radiation. It was also warning that only $72 \%$ of respondent technologists use to wear lead apron during their work. It is due to lack of awareness among professionals and patients regarding the potential harmful effects of unintended medical exposure. These may be caused by insufficient knowledge of the risks and relevant contributing factors among medical professionals, regulatory and public health authorities. 


\begin{abstract}
Justification of the study:
Radiology technologist should always adhere to radiation protection/safety protocols in their daily practices, they could protect themselves and patients from harmful effects of ionizing radiation.
\end{abstract}

\section{Research ouestion:}

How much knowledge and awareness does a radiology technologist have to protect himself, patient, public and environment from ionizing radiation (x-ray)?

Objective:

Hospitals are medical institutions that contain a lot of risk factors. One of these factors is that employees, general public and patients are exposed to ionizing radiation. The purpose of this study was to examine the knowledge and awareness level of radiology technologist regarding radiation safety and to emphasize the level of knowledge towards radiation safety.

Copy Right, IJAR, 2019,. All rights reserved.

\section{Introduction:-}

Ionizing radiation in medical imaging is one of the powerful diagnostic tools in medicine. But this type of radiation has hazardous effects on biological systems. They produce some type of injury that is incurable. Although all medical interventions has potential benefits, but it's potential risks should not be ignored. Radiation protection is the science and art of protecting people and the environment from the harmful effects of ionizing radiation. It is also described as all activities directed towards minimizing radiation exposure of patients and personnel during x-ray exposure.[1] In developing nations, more recent studies show that about 3.6 billion imaging studies per year are carried out world-wide, leading to an increase of $70 \%$ in worldwide collective effective dose for medical diagnostic procedures.[2] More concern has recently been appeared in recent studies that the knowledge of referring doctors about radiation doses incurred during diagnostic radiological procedures is deficient [3]. Such information may be of particular relevance when the expansion of imaging technology is considered

The ultimate goal of radiation protection in health care is the safety of radiology technologist, patients and others by minimizing the risks associated with the use of radiation while maximizing benefits for patients' care.[4] The cancer risks arise with radiation have been known. Ionizing radiation may effects on gastrointestinal system, central nervous system, gonads or even whole body. These effects may appear as a somatic effects or in next generation as a genetic effects. So occupational radiation protection is necessary whenever radiation is used in the practice of medicine. Occupational radiation protection measures are necessary for all individuals who work in the diagnostic imaging departments. This includes not only technologists and nurses, but also individuals who may be in a radiation environment only occasionally. All of these individuals may be considered radiation workers, depending on their level of exposure and on national regulations. All workers require appropriate monitoring continuously by common personnel dosimeters like film badge and thermo luminescence dosimeter. They must also receive education and training appropriate to their jobs and take protection by tools and equipment. The amount of absorbed dose is related to exposure factors such as $\mathrm{kV} /$ potential difference and $\mathrm{mA} /$ intensity of the beam and time. Personnel protection device, working in the safe construction decrease personnel exposure dose. Moreover development and refinement of basic safety standards has a great important role to protect radiology staffs. The level of awareness concerning with radiation protection influences in staff behavior. If they have not enough information related to mentioned issue, their action will not be safe and resulted to adverse effects. The aim of this study was to assess the knowledge and attitude of radiologic technologist towards radiation protection.

\section{Material and methods:-}

This is a preliminary descriptive cross sectional study among 75 radiology technologists working in various hospitals in Dhaka city of Bangladesh. The study was conducted through a well-structured self-administered questionnaire consisting of four parts:

1. First Part: Socio- Demographic data (age, sex, level of education and work experiences).

2. Second Part: Knowledge regarding radiation protection (9 question).

3. Third Part: Performance towards radiation safety (8question). 
4. Fourth Part: Attitude towards the radiation safety (1 Question).

Explanation about the objectives of the study and the benefit of its findings to radiology technologist was provided to each study participant before submitting the questionnaire. Then questionnaire forms were directly distributed to all radiology technologists who work in various hospitals and only 75 radiology technologists participated in this study.

Knowledge was assessed based on study participants understanding on radiation risks associated with diagnostic use of ionizing radiation to protect themselves as well as patient from risks. Radiation protection awareness was assessed by the use of radiation signs during exposures times, using of protective equipment's during work such as lead shield, gonad shields, thyroid cola, lead gloves and light beam diaphragm (LBD). Knowledge was assumed to be poor in this study if respondents' average score is below 05 (Five) out of 09(nine) given in table (2).

\section{Ethical considerations:}

All participants were consented verbally to fill the questionnaires and join the study and no names or any personal data were available to publish.

\section{Results:-}

Table 1: Demographic data of participants $\mathrm{N}=75$

\begin{tabular}{|l|l|l|l|}
\hline Demographic Data & Variable & Number & Percentage \\
\hline \multirow{5}{*}{ Age } & $18-25$ Years & $\mathbf{3 0}$ & $\mathbf{4 0 \%}$ \\
\cline { 2 - 4 } & $\mathbf{2 6 - 3 5}$ Years & $\mathbf{3 5}$ & $\mathbf{4 6 \%}$ \\
\cline { 2 - 4 } & $\mathbf{3 6 - 4 5}$ Years & $\mathbf{8}$ & $\mathbf{1 1 \%}$ \\
\cline { 2 - 4 } & $\mathbf{4 6 - 6 0}$ Years & $\mathbf{2}$ & $\mathbf{3 \%}$ \\
\hline \multirow{5}{*}{ Educational Qualification } & Diploma & $\mathbf{6 0}$ & $\mathbf{8 0 \%}$ \\
\cline { 2 - 4 } & B.Sc. & $\mathbf{1 5}$ & $\mathbf{2 0 \%}$ \\
\hline & $1-5$ Years & $\mathbf{4 0}$ & $\mathbf{5 3 \%}$ \\
\cline { 2 - 4 } & $6-10$ Years & $\mathbf{1 9}$ & $\mathbf{2 5 \%}$ \\
\cline { 2 - 4 } & $11-16$ Years & $\mathbf{1 1}$ & $\mathbf{1 4 \%}$ \\
\cline { 2 - 3 } & $17-$ more & $\mathbf{5}$ & $\mathbf{7 \%}$ \\
\hline
\end{tabular}

Table (1) demonstrates that most of participants were from age group (26-35Y). In education most of them are Diploma holders $(80 \%)$ and $53 \%$ has working experience 1 to 5 years.

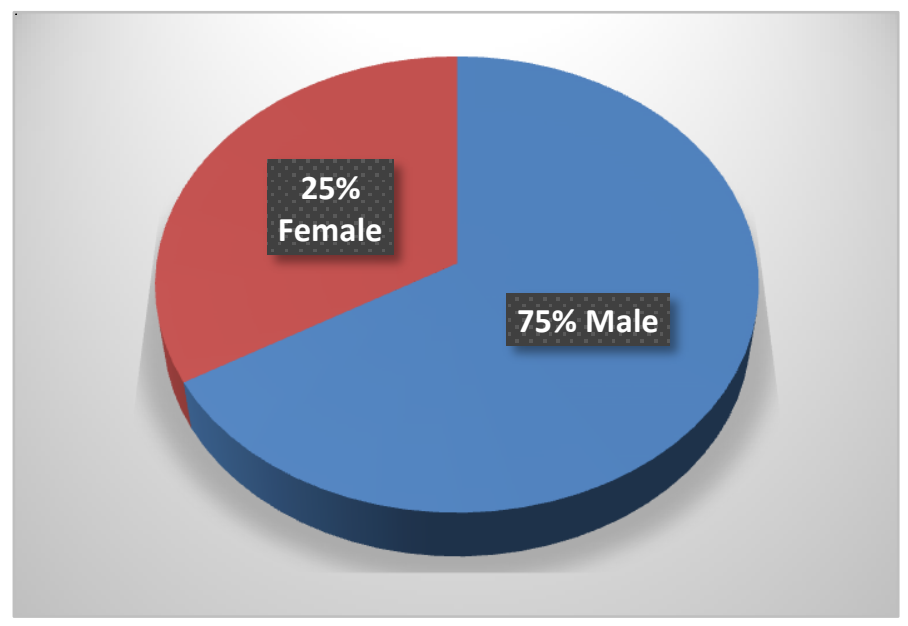

Fig 1:-Distribution of participant's sex wise

Table 2:-Knowledge of participants regarding protection during practice $\mathrm{N}=75$

\begin{tabular}{|l|l|l|}
\hline Variable & Number & $\begin{array}{l}\text { Correct answer } \\
(\boldsymbol{\%})\end{array}$ \\
\hline Thickness of doors and walls of x-ray room for more protection. & $\mathbf{5 0}$ & $\mathbf{6 6 . 6 6 \%}$ \\
\hline
\end{tabular}




\begin{tabular}{|l|l|l|}
\hline Distance of control panel from x-ray source. & $\mathbf{2 5}$ & $\mathbf{3 3 \%}$ \\
\hline Thickness of lead glass and lead apron. & $\mathbf{1 0}$ & $\mathbf{1 3 \%}$ \\
\hline Knowing annual limitation dose for individuals. & $\mathbf{1 0}$ & $\mathbf{1 3 \%}$ \\
\hline You know dosimeter & $\mathbf{3 0}$ & $\mathbf{4 0 \%}$ \\
\hline Knowing ionizing radiation & $\mathbf{1 0}$ & $\mathbf{1 3 \%}$ \\
\hline You know "BAERA" & $\mathbf{2 0}$ & $\mathbf{2 7 \%}$ \\
\hline You Know "ALARA" & $\mathbf{1 0}$ & $\mathbf{1 3 \%}$ \\
\hline How to use TLD batch? & $\mathbf{2 5}$ \\
$\quad-\quad$ TLD wearing is inside or outside lead apron? & \\
$-\quad$ Which is the correct place to wearing TLD batch? & & \\
$-\quad$ Which side of TLD batch placed anteriorly? & & \\
\hline
\end{tabular}

From Table 2 it was revealed that although Radiology Technologist have good knowledge on room protection, comparatively they have very poor knowledge on ionizing radiation, Atomic regulatory board, ALARA principle, even on personal dosimeter. Although we know how much essential to have all knowledge regarding the abovementioned topics for a radiology technologist.

\section{Level of knowledge:}

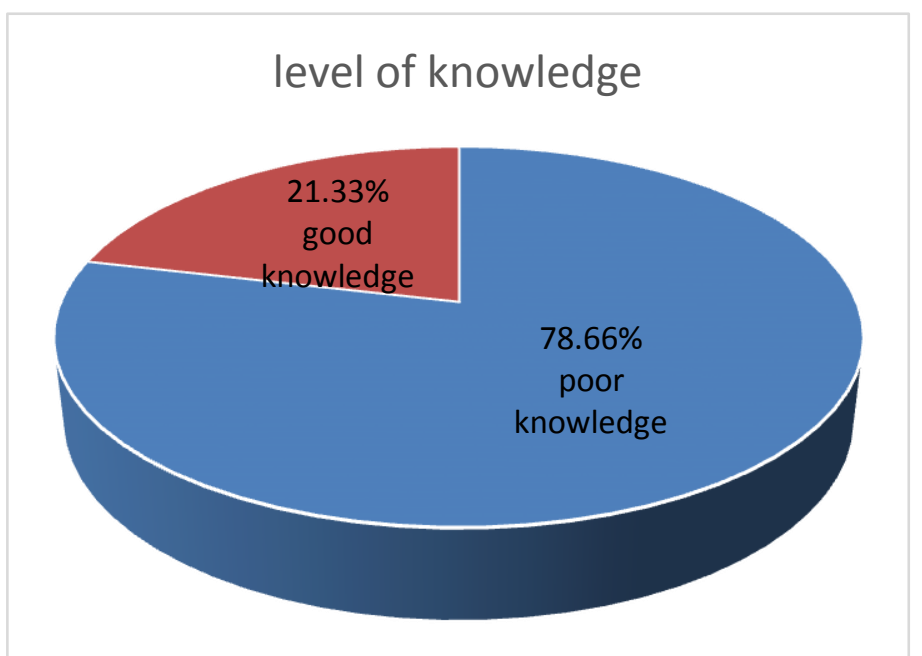

Fig 2:-Distribution of knowledge level of Radiology Technologist regarding radiation protection and principle

From the study sample only $21.33 \%$ have good knowledge regarding radiation protection, and it can be justified that as most of technologist were from Diploma holders, education level is a great factor to have better knowledge.

Table 3:-Performance and awareness of participants toward protection during practices $\mathbf{N}=\mathbf{7 5}$

\begin{tabular}{|l|l|l|}
\hline Variable & Number & Percentage \\
\hline Wearing TLD daily & $\mathbf{3 0}$ & $\mathbf{4 0 \%}$ \\
\hline Wearing lead apron during working hours & $\mathbf{0 5}$ & $\mathbf{6 . 6 6 \%}$ \\
\hline Using light beam diaphragm, cone and grid in every exposure & $\mathbf{5 0}$ & $\mathbf{6 6 . 6 6 \%}$ \\
\hline Using lead gloves during work & $\mathbf{0 0}$ & $\mathbf{0 0 \%}$ \\
\hline Using wall shield or control panel during work & $\mathbf{7 0}$ & $\mathbf{9 3 . 3 3 \%}$ \\
\hline Using Radiation signs or red light during working hours & $\mathbf{3 0}$ & $\mathbf{4 0 \%}$ \\
\hline Wearing thyroid cola during work & $\mathbf{2 5}$ & $\mathbf{3 3 . 3 3 \%}$ \\
\hline Wearing Gonad Shield during work & $\mathbf{0 0}$ & $\mathbf{0 0 \%}$ \\
\hline $\begin{array}{l}\text { Using mechanical or immobilization device for child and old } \\
\text { patient }\end{array}$ & $\mathbf{0 0}$ & $\mathbf{0 0 \%}$ \\
\hline Provide any shielding or lead apron to the patients helper & $\mathbf{1 0}$ & $\mathbf{1 3 . 3 3 \%}$ \\
\hline $\begin{array}{l}\text { Covering all of body with lead apron without the region of } \\
\text { interest }\end{array}$ & $\mathbf{0 3}$ & $\mathbf{0 4 \%}$ \\
\hline
\end{tabular}


From Table 3 it is noticed that among radiology technologists, the practice of using lead gloves, mechanical or immobilization device, Gonad Shield are very poor. In other words it can be said that they have lack of knowledge regarding the importance of wearing the above mentioned radiation protection device. Moreover most of radiology technologists are not aware in safety maintenance in case of patient and patient attendant. Here education level and proper training may be factors and also authorities should monitor them regularly.

Table 4:-Attitude and belief about Ionizing Radiation ( $\mathrm{N}=75)$ According to knowledge and experience what do you think about Ionizing Radiation (x-ray)?

\begin{tabular}{|l|l|l|}
\hline Very dangerous & Dangerous & No dangerous \\
\hline $\mathbf{5 0 \%}$ & $\mathbf{5 0 \%}$ & Nil \\
\hline
\end{tabular}

\section{Discussion:-}

Medical Technology is an emerging field for career building across the world. In this preliminary descriptive study awareness was assessed by measures knowledge of radiology technologist towards radiation safety during practice in Dhaka city of Bangladesh. A total of 75 radiology technologist responded to this study, from them there were (75\%) male and (25\%) female, their ages ranged between 20 year and 60 years, most of them are Diploma holders $(80.7 \%)$ in education. Though the collection of data did not find any relation between level of education of participants and work expertise with their knowledge around necessity performance of periodical examination and also application of organ shield for patients and themselves, education level and proper training may be factors. In addition application of personal dosimeter was not affected by age, level of education and also work experience of participants. It is just not taking it seriously by the technologists.

The working experience of the participants in this study ranged between one year and 40 years. According to data analysis, there was no significant relation between awareness of radiation safety, performance and work experience. Regarding knowledge in this study (66.66\%) of the respondents knew that doors and walls consist of isolated materials such as lead. About $40.7 \%$ wearing TLDs during their work hours and 15-20\% participants said they are not regular wearing TLD batch. About $40 \%$ participant did not have TLD batch. In this study and responded to the question about amount of annual dose limit for individuals and data analysis show that the majority of workers had not correct answer (more than 75\%). Knowledge has been assumed to be poor in this study if respondents' average score on nine questions used to assess knowledge is less than five correct answers, and according to the result (21.3\%) had good knowledge regarding protection, fig (1). 6.66\% of the participants in this study wearing lead apron during work because they said during the exposure they are staying behind a protective barrier during their work time. They think that protective barrier in front of control panel is enough to avoid scattered radiation. It was also informed that sufficient number of lead apron is not available in the departments and also there were no gonad shield and lead gloves at all. Using of light beam diaphragm and other protective devices (cone \& grid) have percentage of $(66.66 \%)$, radiation signs during working hours with $(40 \%)$.

Thyroid protective shield are used by (33.3\%) in this study only during fluoroscopy study, while many investigations have been done and clearly demonstrated the efficacy of protection equipment and the importance of shielding the radiation-sensitive organs for reducing the absorbed dose. [9] [10]

Protection (ICRP) radiation safety standards, for gonads shields to be used for the protection of the gonads when the pelvis is not part of the anatomical area being examined.[11] The gonad area must be shielded whenever in a primary radiation field or very close to a primary radiation field. Most of participant told me they did not have any mechanical/immobilization device in their department to reduce the repeat $\mathrm{x}$-ray as well as radiation dose to the patient and attendance. They did not use to provide lead apron to the attendance during assistance or help to the patient unless they want.

\section{Conclusion:-}

In this study it may be concluded that following three factors are the reason behind the unawareness of radiology technologist regarding radiation safety principle:

1. Lack of adequate knowledge towards radiation safety,

2. Lack of supervision of concern authority,

3. Harmful effect by x-ray is not observed in naked eye instantaneously during most of imaging diagnosis. 


\section{Suggestion for future study:}

It is important for all radiology technologists to continue professional development; by holding more workshops, short-term training courses, preparation and distribution of posters on the protection and safety against ionizing radiation in order to raise knowledge and performance to include the most recent trends in radiation protection. Similar studies with larger sample size at regular intervals should be carried out for strict adherence of standard radiation protection regulation protocol.

\section{References:-}

1. Johnston J, Killion JB, Veale B, Comello R. U.S. technologists' radiation exposure perceptions and practices. Radiol Technol. 2011; 82: 311-20.

2. The International Commission on Radiological Protection; Annuals of the ICRP 2011. Draft report on radiological protection in fluoroscopically guided procedures performed outside the imaging department. Available from http: //www. icrp. org/ docs /Radiological protection in fluoroscopically guided procedures performed outside the imaging department, pdf.

3. Gower-Thomas K, Lewis MH, Shiralkar S, Snow M, Galland RB, Rennie A. Doctor's knowledge of radiation exposures is deficient. Br Med $\mathrm{J}$ 2002;324:919.

4. www.who.int/ionizing_radiation/pub_meet/chapter2. pdf, Chapter 2: Radiation protection concepts and principles

5. Rania Mohammed Ahmed, Afaf Mohamed Taha Elamin, Elsamani M , Wisal B Hassan, "Knowledge and Performance of Radiographers towards Radiation Protection, Taif, Saudi Arabia" IOSR Journal of Dental and Medical Sciences (IOSR-JDMS). e-ISSN: 2279-0853, p-ISSN: 2279-0861.Volume 14, Issue 3 Ver. II (Mar. 2015)

6. Maryam Mojiri , Abbas Moghimbeigi, "Awareness and attitude of radiographers towards radiation protection", Journal of Paramedical Sciences (JPS) Autumn 2011 Vol.2, No.4 ISSN 2008-4978

7. European Commission. Radiation protection 116. Guidelines on education and training in radiation protection for medical exposures. Luxembourg: European Commission. Directorate-General for the Environment , 2000. Available at: http://ec.europa.eu/energy /nuclear/ radiation_ protection/doc/publication/116.pdf. Accessed August 16, 2009.

8. T.R. Goodman, MD "Ionizing Radiation Effects and Their Risk to Humans"Yale University School of Medicine, New Haven, CT .

9. Borhani P, Alizade M .[Evaluation of radiology personnel practice of Kerman university of medical science hospitals]. Med J Hormozgan Univ 2003; 6(4):51-8.

10. Foley SJ, McEntee MF, Achenbach S, Brennan PC, Rainford LS, Dodd JD. Breast surface radiation dose during coronary CT angiography: reduction by breast displacement and lead shielding. AJR Am J Roentgenol 2011;197(2):367-73.

11. Persson L, Shrader-Frechette K. An evaluation of the ethical principles of the ICRP's radiation protection standards for workers. Health physics 2001, 80, $3: 225-234$

12. Karen E, Thomas.June E, Parnell-Parmley, Haidar S, Moineddin R et al. Assessment of radiation dose awareness among pediatricians. Pediatr Radiol 2006, 36: 823-832

13. Kaushal V, Joshi CP, Passi K,Maria Das J, Aggarwal L, Kheruka S, Kaushal J. Occupational exposure to staff of a major radiological center-trend in radiation safety. Journal of Medical physics 1990, 15(2): 141-143.

14. Amirzadeh F, Tabatabaee SHR. Evaluation of healthy behavior in radiation employees in hospitals of Shiraz. The fourth international cyberspace conference on ergonomics. International ergonomics association press 2005. 